\title{
Experimental Therapeutics for Dystonia
}

\author{
H. A. Jinnah* and Ellen J. Hess*广 \\ Departments of $*$ Neurology and ${ }^{\dagger}$ Neuroscience, Johns Hopkins University School of Medicine, Baltimore, Maryland 21287
}

Summary: Dystonia is a neurological syndrome characterized by excessive involuntary muscle contractions leading to twisting movements and unnatural postures. It has many different clinical manifestations, and many different causes. More than 3 million people worldwide suffer from dystonia, yet there are few broadly effective treatments. In the past decade, progress in research has advanced our understanding of the pathogenesis of dystonia to a point where drug discovery efforts are now feasible. Several strategies can be used to develop novel therapeutics for dystonia. Existing therapies have only modest efficacy, but may be refined and improved to increase benefits while reducing side effects. Identifying rational targets for drug intervention based on the pathogenesis of dystonia is another strategy. The surge in both basic and clinical research discoveries has provided insights at all levels, including etiological, physiological and nosological, to enable such a targeted approach. The empirical approach to drug discovery, whereby compounds are identified using a nonmechanistic strategy, is complementary to the rational approach. With the recent development of multiple animal models of dystonia, it is now possible to develop assays and perform drug screens on vast numbers of compounds. This multifaceted approach to drug discovery in dystonia will likely provide lead compounds that can then be translated for clinical use. Key Words: Dystonia, animal models, drug discovery, pathogenesis, therapy.

\section{DEFINITION AND CLASSIFICATION OF THE DYSTONIAS}

Dystonia is a neurological syndrome in which the fundamental problem involves excessive involuntary muscle contractions. ${ }^{1,2}$ Muscles involved in an intended movement contract excessively, and there is overflow contraction of nearby muscles not needed for the movement. The overflow sometimes includes muscles that oppose the primary muscles, leading to co-contraction of agonist and antagonist muscles. The same muscles tend to be involved in an individual, leading to repetitive and patterned movements.

The appearance of the movements depends on the strengths and combinations of the muscles involved. In its mildest form, dystonia may appear merely as an exaggeration of an otherwise normal movement. In more severe forms, dystonia is expressed as movements that are stiff, slow, twisting, or jerky. In its most severe forms, dystonia is characterized by unnatural postures or fixed deformities. The many different overt clinical man-

Address correspondence and reprint requests to: Ellen J. Hess, Ph.D., Department of Neurology, Meyer Room 6-181, Johns Hopkins University, $600 \mathrm{~N}$. Wolfe Street, Baltimore, MD 21287. E-mail: ehess@jhmi.edu. ifestations are classified according to the parts or extent of the body affected:

- Focal dystonias, involving an isolated body region Blepharospasm (periocular muscles only)

Cervical (torticollis)

Laryngeal (spasmodic dysphonia)

Oromandibular (jaw, tongue, or perioral)

Limb (writer's cramp, foot dystonia)

- Segmental dystonias, involving two or more contiguous regions

Meige syndrome (blepharospasm and oromandibular dystonia)

Cervical dystonia and one arm

- Multifocal dystonias, involving two or more noncontiguous regions

- Hemidystonia, involving half of the body (e.g., ipsilateral arm and leg)

- Generalized dystonias, with broader involvement (including at least one leg)

Both legs and another region, with or without the trunk

One leg, another region, and trunk

The dystonias also can be classified according to the presence or absence of accompanying features. In the 
primary dystonias, accompanying problems are absent or minimal, and dystonic movements occur in relative isolation. The dystonia-plus syndromes include a select group of disorders in which dystonia is accompanied by specific neurological features. The secondary dystonias include a broad group of disorders in which dystonia is due to specific identifiable causes, either inherited or acquired (Table 1). Dystonia can be secondary to focal lesions of the nervous system, toxins, medications, metabolic disturbances, infectious or inflammatory conditions, and developmental or degenerative diseases.

\section{EPIDEMIOLOGY OF THE DYSTONIAS}

Good estimates for the incidence and prevalence of the dystonias are limited by the lack of comprehensive epidemiological studies. Studies of primary dystonia have estimated prevalence to be approximately 370 per 1 million people. ${ }^{3}$ These estimates imply that primary dystonia affects more than 3 million people in the world; however, it is widely believed that these numbers are underestimates, because the many different clinical manifestations of primary dystonia are not well recognized and many patients are not diagnosed.

The numbers of people affected with secondary dystonias are even less well characterized. Many of the hereditary metabolic disorders associated with dystonia are rare, with a prevalence of only a few cases per million. Other secondary dystonias are more common. For example, cerebral palsy occurs with an incidence of 1.5 per 1000 live births per year. Approximately onethird of affected children exhibit dystonia, and in some it is the major source of motor disability. ${ }^{4-6}$ Parkinson's disease has a prevalence of $1 \%$ among people over 65 years of age. Approximately one-third suffer from dystonic movements at some point in their illness, either as a presenting feature or a complication of therapy., Thus, it is useful to consider all populations, both primary and secondary, that may benefit from the development of novel therapeutics. If novel therapeutics target both primary and secondary dystonias, they have the potential to benefit large numbers of people.

\section{CURRENT TREATMENT OPTIONS}

Given several excellent recent reviews, ${ }^{9-12}$ current treatments are summarized only briefly here. Few treatments have broad efficacy in the dystonias. The botulinum toxins are the most broadly effective. They must be injected into affected muscles, so they are most useful in the focal and segmental dystonias, where a limited number of muscles can be targeted. Botulinum toxins can also be useful in suppressing the most troublesome features in patients with broader involvement, including the generalized dystonias, but injections into all affected muscles are not practical. Benefits from the injections last for 3 to 4 months, so they are repeated several times yearly. Resistance develops in a small proportion of patients with repeated treatment.

Anticholinergics also can be quite helpful in some patients, although relatively high doses are required..$^{9-12}$ The best responses are reported in selected children with generalized or segmental dystonia; many children, however, do not respond. Efficacy in adults is less dramatic, and more frequently limited by side effects. Because relatively high doses are required, side effects become increasingly bothersome as doses increase. These effects include cognitive dulling, short-term memory impairment, dry mouth, constipation, and urinary retention, among others.

Other medications are useful for specific subtypes of dystonia. Children with dopa-responsive dystonia, which is caused by defects in dopamine synthesis, exhibit excellent responses to the dopamine precursor levodopa. Levodopa also is useful for dystonic manifestations in Parkinson's disease, and occasionally is effective in other childhood or adult dystonias. It is not useful in the majority of dystonias, however.

Suppressing dopamine transmission also is reported to be beneficial. Depletion of dopamine with tetrabenazine can be helpful in some dystonias, particularly tardive dystonias. Dopamine receptor antagonists are sometimes offered, but they are not widely used, because they cause side effects (such as tardive syndromes or parkinsonism) that can introduce diagnostic confusion with the disorder being treated.

Several other medications are sometimes recommended for use in dystonia. These include baclofen, benzodiazepines, carisoprodol, cyclobenzaprine, methocarbamol, metaxalone, mexiletine, and tizanidine, among others. These medications seem to be at least partly helpful in some cases, although rigorous placebo-controlled studies with adequate statistical power to guide expectations for specific subgroups are lacking.

Surgical interventions have become increasingly popular in the face of limited efficacy of medical treatments. Because of the associated risks, however, surgical procedures are typically reserved until other less-invasive options have proven unsatisfactory. Selective peripheral denervation can be offered to patients with cervical dystonia. ${ }^{13}$ Intrathecal baclofen can be helpful in selected populations. ${ }^{6,14}$ Interest in deep brain stimulation in particular has been growing, and it is the focus of another review in this issue. ${ }^{15}$

\section{EXPERIMENTAL STRATEGIES FOR NEW TREATMENTS}

The relative scarcity of broadly effective medical treatments, together with the combined prevalence of primary 
TABLE 1. Causes for Dystonia

\begin{tabular}{|c|c|}
\hline Inherited Dystonia & Acquired or Sporadic Dystonia \\
\hline $\begin{array}{l}\text { Amino acid metabolism } \\
\text { glutaric acidemia } \\
\text { GAMT deficiency } \\
\text { Hartnup disease } \\
\text { homocystinuria } \\
\text { methylmalonic acidemia } \\
\text { propionic acidemia } \\
\text { sulfite oxidase deficiency } \\
\text { Neurotransmitter metabolism } \\
\text { AADC deficiency } \\
\text { dihydropterin reductase deficiency } \\
\text { GTP cyclohydrolase deficiency } \\
\text { PTPS deficiency } \\
\text { tyrosine hydroxylase deficiency } \\
\text { Lipid metabolism/storage } \\
\text { GM1 or GM2 gangliosidosis } \\
\text { Krabbe's disease } \\
\text { metachromatic leukodystrophy } \\
\text { neuronal ceroid lipofuscinosis } \\
\text { Niemann-Pick disease, type C } \\
\text { Pelizaeus-Merzbacher disease } \\
\text { Ion/metal homeostasis } \\
\text { aceruloplasminemia } \\
\text { Cav2.1 calcium channel defects } \\
\text { Fahr disease } \\
\text { neuroferritinopathy } \\
\text { rapid-onset dystonia-parkinsonism } \\
\text { Wilson's disease } \\
\text { Polyglutamine expansions } \\
\text { dentatorubral-pallidoluysian atrophy } \\
\text { Huntington's disease } \\
\text { spinocerebellar ataxias (1, 2, 3, 6, 7, 17) } \\
\text { DNA handling and transcription } \\
\text { ataxia-oculomotor apraxia } \\
\text { ataxia telangiectasia } \\
\text { Cockayne's syndrome } \\
\text { Lubag } \\
\text { Rett syndrome } \\
\text { xeroderma pigmentosum } \\
\text { Mitochondrial function } \\
\text { dystonia-deafness syndrome } \\
\text { fumarase deficiency } \\
\text { Leber's hereditary optic neuropathy } \\
\text { Leigh disease } \\
\text { MELAS } \\
\text { MERRF } \\
\text { pyruvate dehydrogenase deficiency } \\
\text { Other } \\
\text { ataxia with vitamin E deficiency } \\
\text { biotin responsive basal ganglia disease } \\
\text { frontotemporal dementias } \\
\text { Lesch-Nyhan disease } \\
\text { myoclonus dystonia } \\
\text { neuroacanthocytosis } \\
\text { neuronal intranuclear inclusion disease } \\
\text { Oppenheim dystonia } \\
\text { pantothenate kinase neurodegeneration } \\
\text { triosephosphate isomerase }\end{array}$ & $\begin{array}{l}\text { Medications } \\
\text { carbamazepine } \\
\text { cinnarizine } \\
\text { dopamine antagonists or agonists } \\
\text { fenfluramine } \\
\text { flunarizine } \\
\text { levodopa } \\
\text { phenytoin } \\
\text { prochlorperazine } \\
\text { metoclopramide } \\
\text { serotonin uptake inhibitors } \\
\text { tiagabine } \\
\text { Toxins } \\
\text { 3-nitropropionic acid } \\
\text { bilirubin (kernicterus) } \\
\text { carbon disulfide } \\
\text { carbon monoxide } \\
\text { cyanide } \\
\text { disulfiram } \\
\text { manganese } \\
\text { methanol } \\
\text { Vascular } \\
\text { stroke (hemorrhagic or ischemic) } \\
\text { vascular malformation } \\
\text { vasculitis } \\
\text { Infection } \\
\text { bacterial } \\
\text { fungal } \\
\text { prion } \\
\text { protozoan } \\
\text { viral } \\
\text { Autoimmune } \\
\text { antiphospholipid syndrome } \\
\text { dystonia gravidarum } \\
\text { hymenoptera stings } \\
\text { multiple sclerosis } \\
\text { Reye's syndrome } \\
\text { Sjögren's syndrome } \\
\text { systemic lupus erythematosus } \\
\text { subacute sclerosis panencephalitis } \\
\text { Trauma } \\
\text { head } \\
\text { nerve } \\
\text { spine } \\
\text { Structural } \\
\text { abscess } \\
\text { Arnold-Chiari malformation } \\
\text { atlanto-axial subluxation } \\
\text { syringomyelia } \\
\text { tumors (brain, spine) } \\
\text { Other } \\
\text { cerebral palsy } \\
\text { corticobasal ganglionic degeneration } \\
\text { hypoparathyroidism } \\
\text { multiple system atrophy } \\
\text { Parkinson's disease } \\
\text { progressive supranuclear palsy } \\
\text { tic disorders } \\
\text { and }\end{array}$ \\
\hline
\end{tabular}

Although tabulation of dystonia causes commonly is organized according to mode of inheritance, this table is organized according to biochemical or functional defects, to emphasize the concept of shared themes in pathogenesis.

$\mathrm{AADC}=$ aromatic amino acid decarboxylase; Cav2.1 = P/Q-type voltage regulated calcium channel; GAMT = guanidinoacetate methyltransferase; GTP = guanosine triphosphate; MELAS = mitochondrial encephalopathy with lactic acidosis and stroke-like episodes; MERRF = mitochondrial encephalopathy with ragged red fibers; PTPS = pyruvoyltetrahydropterin synthase; SCA = spinocerebellar ataxia.

and secondary dystonias, provides a great opportunity for experimental therapeutics. There currently are several strategies that are being pursued to develop new treatments.
These include improving upon existing therapies, elucidation of pathogenesis to identify new targets for intervention, and empirical screens in preclinical models. 


\section{Improve existing therapies}

One strategy for developing new treatments is to improve upon existing ones. The relatively broad, but often modest, efficacy of anticholinergics in both primary and secondary dystonias provides an obvious candidate for further refinement. The mechanism of action of anticholinergics in dystonia is not completely understood, but there has been an enormous increase in our understanding of acetylcholine neurotransmission and its relevance to movement disorders. ${ }^{16}$ This new information can be exploited to refine the benefits of anticholinergics and to reduce the nonspecific side effects that often limit the use of these drugs.

There are several cholinergic pathways in the brain. Those considered relevant to dystonia involve the giant aspiny interneurons of the striatum. These neurons constitute fewer than $1 \%$ of all striatal neurons, but they have broad axonal arbors with dense terminal fields blanketing the entire striatum. Additionally, the majority of their varicosities do not form typical synapses, suggesting a volumetric mode of neurotransmission, characterized by extrasynaptic diffusion of signaling molecules. These interneurons are tonically active, with autonomous pace-making activity. This tonic activity is extrinsically modulated by inputs from neurons that use dopamine, glutamate, or $\gamma$-aminobutyric acid. Acetylcholine released from the giant aspiny interneurons exerts its effects via two classes of receptors: nicotinic and muscarinic. The muscarinic receptors are divided into five subtypes, M1 to M5. Corticostriatal terminals express presynaptic M2 and M3 receptors; the M1 and M4 receptors are found postsynaptically on medium spiny neurons, which are the major striatal projection neuron. The muscarinic receptors are coupled to cell-surface calcium or potassium channels, intracellular calcium mobilization, phospholipase $\mathrm{C}$, or protein kinase $\mathrm{C}$. Acetylcholine has many functional effects on striatal neurons. Of particular relevance to dystonia is the ability of acetylcholine to modulate corticostriatal plasticity, as determined by longlasting changes in the efficiency of synapses between corticostriatal terminals and medium spiny neurons.

The precise mechanisms by which anticholinergics are helpful in dystonia are not fully understood. Further elucidating the responsible mechanisms could provide several rational targets for drug development; these might include drugs that modify the tonic activity of cholinergic interneurons, alter volumetric transmission, more specifically target the relevant muscarinic receptors while reducing effects at nicotinic receptors, influence one of the many receptor-effector mechanisms, or modify the development of plasticity. By more precisely targeting the relevant mechanism, it may be possible to develop drugs with greater efficacy, while at the same time reducing the nonspecific side effects associated with influences on other mechanisms.

\section{Rational design: prototype disorder strategy}

A methodical dissection of the pathogenesis of disease to identify rational targets for drug intervention provides another intuitively attractive approach for identifying new treatments. ${ }^{17}$ For most diseases, pathogenesis begins with some initial insult such as a genetic mutation, exposure to some toxin, or focal brain injury (FIG. 1A). The initial insult causes a cascade of downstream events, which ultimately lead to the overt clinical manifestations of the disease. Elucidating each step in the pathway can provide rational targets for drug intervention.

For example, parkinsonism may begin with an inherited insult affecting the genes encoding LRRK2, Parkin, DJ-1, or Pink1. ${ }^{18,19}$ The genetic mutations cause defects in several basic biochemical processes that may result in abnormal intracellular handling of misfolded proteins, mitochondrial dysfunction, oxidative stress, and chronic inflammation. These processes converge upon nigrostriatal dopamine neurons, with degeneration and subsequent impairment of dopamine neurotransmission. The loss of dopamine neurotransmission alters the physiology of the basal ganglia in a manner that results in overt motor dysfunction. Understanding this cascade of events provides several targets for therapy. These include correction of the original genetic defect via gene therapy, ${ }^{20}$ reversal of the basic biochemical defects for symptomatic benefit or neuroprotection, ${ }^{21}$ replacement of depleted dopamine stores or dopamine neurons, ${ }^{22}$ or interruption of abnormal basal ganglia output via deep brain stimulation. $^{23,24}$

As another example, dopa-responsive dystonia may begin with a mutation in the $G C H I$ gene (FIG. 1B). ${ }^{25}$ The mutation leads to its primary biochemical defect affecting the enzyme GTP-cyclohydrolase. The primary biochemical defect then leads to secondary and tertiary biochemical changes, involving reduced synthesis of tetrahydrobiopterin and dopamine. The deficiency of dopamine subsequently alters the physiology of motor control pathways in the basal ganglia. The abnormal physiology leads to involuntary muscle overcontraction, with twisting movements and abnormal posturing characteristic of dystonia. Understanding this cascade of events provides several targets for therapy. These include correction of the original genetic defect via gene therapy, supplementation of depleted tetrahydrobiopterin or dopamine, correction of the abnormal basal ganglia physiology, or direct treatment of overactive muscles with botulinum toxin. For dopa-responsive dystonia, restoring deficient dopamine stores with levodopa provides the most convenient solution.

A challenge in basing rational drug design on pathogenesis in dystonia involves etiologic heterogeneity. Unlike Parkinson's disease, in which a limited number of initial insults converge upon a relatively focal neurodegenerative process, there are many different etiologies 


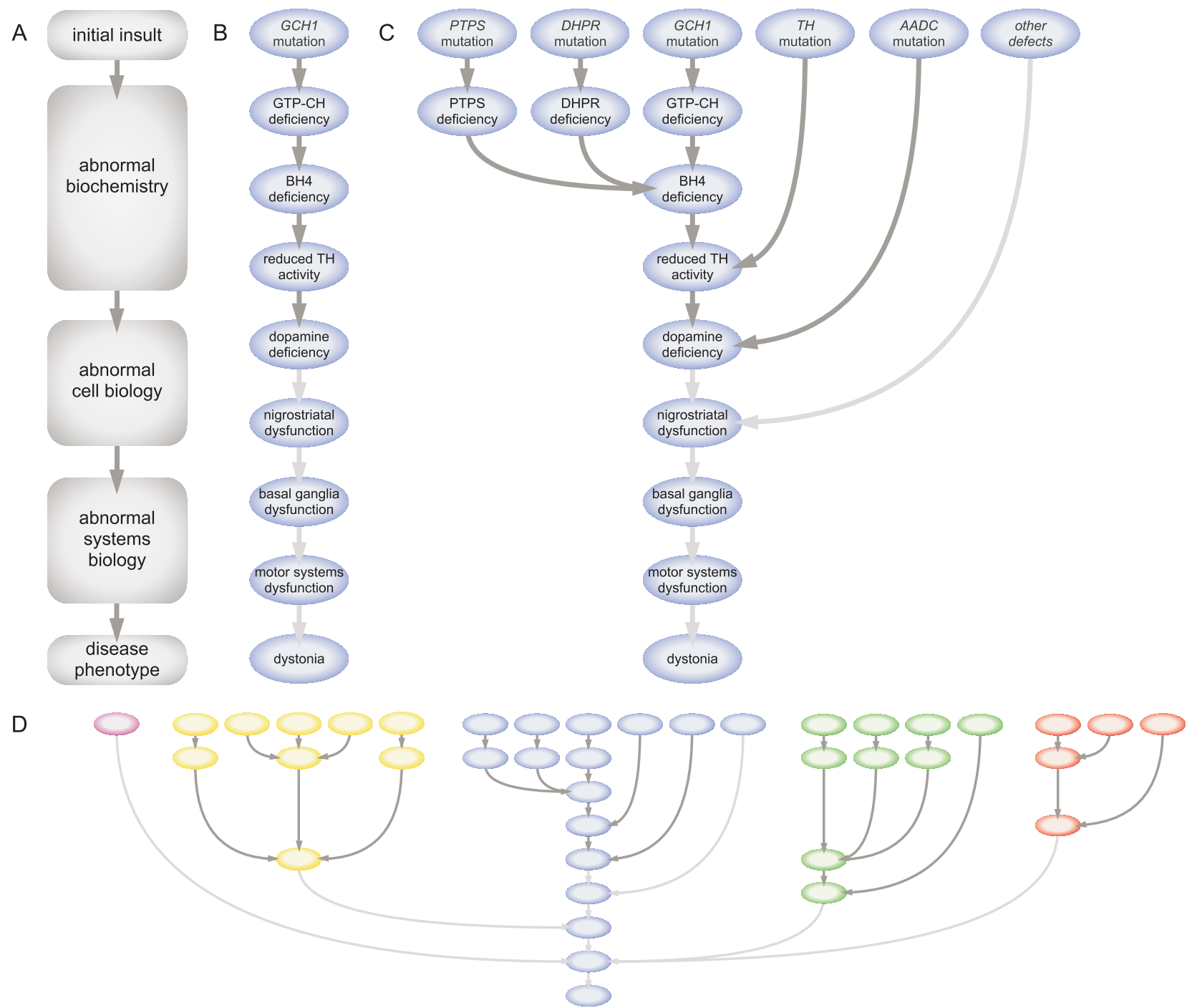

FIG 1. Schematic for drug design based on pathogenesis of dystonia. A: Simplified schematic for the pathogenesis of disease. Pathogenesis proceeds through a cascade of events that include molecular, biochemical, cellular, systems, and functional levels. B: Schematic for pathogenesis of dopa-responsive dystonia beginning with mutation of the GCH1 gene, proceeding through primary (GTP-cyclohydrolase) and secondary (tetrahydrobiopterin [BH4], tyrosine hydroxylase [TH]) biochemical abnormalities, disruption of cell signaling involving dopamine, dysfunction of dopamine-dependent basal ganglia pathways, and abnormal function of the motor circuit causing dystonia. Well-established relationships are shown as dark arrows; presumed or suspected relationships are shown as gray arrows. C: Schematic for other disorders that may share pathogenesis at different biological levels. Other disorders might include Parkinson's disease, acute or chronic drug-induced dystonia, and Lesch-Nyhan disease. DHPR = dihydropteridine reductase; PTPS = 6-pyruvoyltetrahydropterin synthase. D: Schematic that accounts for many other etiologies for dystonia, depicting the likelihood that some subgroups are likely to share specific biochemical or physiological mechanisms. Appreciating the complex pathogenesis of the dystonias is important for identifying rational biological targets for drug intervention.

and pathological processes in dystonia. Inherited causes for dystonia include nearly all basic cellular processes, including amino acid metabolism, organic acid metabolism, lipid metabolism, purine metabolism, protein folding or trafficking, ion homeostasis, neurotransmission, cellular handling of heavy metals, DNA processing, mitochondrial function, and others (Table 1). Acquired cases reveal that relevant pathology may occur in different areas of the nervous system, including the cerebral cortex, basal ganglia, cerebellum, brainstem, spinal cord, and even peripheral nerve. ${ }^{26,27}$ In addition to the heter- ogeneity of biochemical and anatomical substrates, some dystonias result from abnormalities of developmental processes but others are a consequence of degeneration. This remarkably diverse array of etiologies means that the resulting pathogenic processes differ among the dystonias, with the implication that drug targets also will differ. $^{17}$

Two strategies are being pursued to address the challenge of pathogenic heterogeneity in dystonia. The first involves elucidation of pathogenesis in selected prototype disorders, with the hope that findings will generalize 
to other populations. Early-onset primary torsion dystonia, sometimes known as Oppenheim dystonia or DYT1 dystonia (for the $D Y T 1$ gene, since renamed TORIA), has been proposed as a prototype disorder for a number of reasons. One reason is the evidence that the disorder is treatable. At least partial improvements occur with anticholinergic drugs ${ }^{9,10}$ or deep brain stimulation. ${ }^{28}$ The disorder is dominantly inherited with penetrance of only $30 \%$, with most cases arising in childhood. The childhood onset and low penetrance suggest an isolated developmental window of vulnerability that is substantially modified by epigenetic or nongenetic influences. Early manipulation of these processes may be sufficient to prevent onset of disease. Finally, DYT1 dystonia was the first primary torsion dystonia for which the gene product was identified, and the same mutation is found in most cases. ${ }^{29}$ Identification of the gene permits the dissection of initial biochemical pathogenesis, and the genetic uniformity allows for the design of genetic tools that target one mutation or common downstream mechanisms.

DYT1 dystonia is caused by a 3-bp deletion of a $G A G$ codon in the TORIA gene. ${ }^{29}$ The deletion results in the omission of a single glutamate in the protein torsinA. The functions of torsinA are not entirely understood. Based on sequence homology to other proteins, torsinA is believed to be an ATP-dependent molecular chaperone whose function is to facilitate folding of other proteins into their proper three-dimensional structures, or to guide intracellular trafficking of proteins to proper locations within cells, or both. Mutant torsinA is associated with a number of cellular anomalies, including improper protein trafficking, ${ }^{30-32}$ abnormalities in cell secretion or transmitter release, ${ }^{32-34}$ alterations in neuronal architecture or physiology, ${ }^{35,36}$ and perinuclear inclusions. ${ }^{32,37-40}$ At the neuroanatomical level, dystonic movements have been linked with abnormal functioning of a motor network involving the cerebral cortex, basal ganglia, and cerebellum. ${ }^{41}$

Each of the identified pathological processes in DYT1 dystonia could serve as a target for drug therapy. Because it is dominantly inherited, a genetic strategy aimed at reducing expression from the mutant allele could prove useful. One potential genetic approach involves the design of small interfering RNAs that target the product of the mutant allele to increase the proportion of normal RNAs. ${ }^{42}$ Biochemical strategies could involve augmenting the functions of the normal protein product or blocking the functions of the mutant protein. For example, overexpression of normal torsinA may block the formation of deleterious protein aggregates associated with $\alpha$-synuclein or polyglutamine-containing proteins. ${ }^{43-45}$ Deep brain stimulation of the pallidum or thalamus may provide benefits by interrupting abnormal physiological patterns in the motor network responsible for dystonia. ${ }^{15}$
The success of DYT1 dystonia as a prototype for rational drug design depends on two unresolved but important issues. The first is that it is not yet clear which of the many biochemical and cellular consequences of mutant torsin A might be linked to the pathogenesis of dystonic movements. Some of the reported phenomena may represent pleiotropic consequences of the mutation with no relevance to the pathogenesis of dystonic movements. Designing therapies that address the wrong target may do nothing to suppress dystonia. Sorting out which of the biological consequences might be most relevant to the genesis of dystonia is important for guiding selection of biological targets for rational drug design.

The second issue is the risk inherent in using any one disease as a prototype for a broader population with a heterogeneous pathogenesis. ${ }^{17}$ It is not yet clear if work with DYT1 dystonia can provide results relevant to other dystonias. The GAG deletion associated with DYT1 dystonia is infrequently found in more common adult-onset focal dystonias, and some have proposed that its biochemical pathology is unique among the dystonias. ${ }^{30}$ Others have suggested a general relevance to more common dystonias. ${ }^{46}$ For example, some studies have revealed an association between polymorphisms in the TORIA gene and the common adult-onset sporadic focal dystonias. ${ }^{46,47}$ Other studies have not confirmed the associations, ${ }^{48,49}$ or suggested that the associations hold only for certain populations in Iceland and Europe, but not in North America. ${ }^{50}$ The relevance of biochemical pathogenesis in DYT1 dystonia to broader populations of dystonia is important for justifying its use as a prototype disorder for other dystonias, and needs to be resolved.

\section{Rational design: shared pathways strategy}

A second approach for addressing the challenge of pathogenic heterogeneity among the dystonias involves comparing different dystonias to identify shared biological mechanisms that will be relevant to larger subpopulations of patients (FIGS. 1C and 1D). ${ }^{17}$ The shared mechanisms may not accommodate all dystonias, but rather specific clusters. The shared mechanisms may occur at different levels-for example, biochemical or physiological.

One of the earliest shared neurochemical themes to be recognized in dystonia involves defects in dopaminergic neurotransmission. ${ }^{51}$ Dystonia occurs in several inherited defects of dopamine synthesis, such as DOPA-responsive dystonia ${ }^{25}$ or Lesch-Nyhan disease. ${ }^{52,53}$ Dystonia may occur also as an early presenting feature of dopamine neuron loss in Parkinson's disease or as a late consequence of dopaminergic therapies. ${ }^{7,8}$ The early and late dystonic manifestations associated with dopamine neuron loss can be replicated in nonhuman primates exposed to 1-methyl-4-phenyl-1,2,3,6-tetrahydropyridine (MPTP). ${ }^{54,55}$ Imaging $^{56}$ and post mortem studies ${ }^{57}$ have 
revealed subtle abnormalities of dopamine systems in both inherited and sporadic primary dystonias. Even among neurologically normal people, drugs that block dopamine transmission can induce acute dystonic reactions or tardive dystonia. ${ }^{58}$ Abnormalities of dopamine neurotransmission have thus been linked with many different forms of dystonia, suggesting a common neurochemical link. The exact nature of the defect remains uncertain, because drugs that globally enhance or suppress dopaminergic transmission are not broadly effective as treatments. Further elucidating the manner in which dopamine dysfunction results in dystonia may provide a shared biological target for therapeutic intervention that could be effective in several different forms of dystonia.

Another shared theme for a different group of dystonias involves defects in mitochondrial function. ${ }^{59}$ Dystonia can be a prominent feature in inherited mitochondrial disorders such as Leber's optic neuropathy, Leigh disease, or the Mohr-Tranebjaerg dystonia-deafness syndrome (Table 1). In many of these cases, the dystonia is generalized, accompanied by other neurological defects, and associated with overt neuropathological damage in the basal ganglia. In some cases, dystonia can be the presenting problem, ${ }^{60,61}$ the predominant neurological problem, ${ }^{62,63}$ or limited to focal or segmental patterns. ${ }^{63-66}$ Among less severely affected patients, overt neuropathological damage may be absent. These atypical cases may be due to mutations with less severe consequences than those of more classical cases, or to variable penetrance caused by the phenomenon of heteroplasmy in mitochondrial disease.

In addition to inherited mitochondrial diseases, dystonia is a prominent feature among children ${ }^{67}$ and nonhuman primates exposed to the mitochondrial mycotoxin, 3-nitropropionic acid. ${ }^{68}$ Furthermore, functional defects in mitochondrial complex I have been associated with sporadic adult-onset primary focal dystonias. ${ }^{69,70}$ The severity of the defect in complex I seems to correlate with the severity of dystonia, because those with segmental patterns have lower complex I activity than those with focal patterns. Thus, mitochondrial defects are apparent in both inherited and sporadic dystonias of many types, suggesting a common subcellular defect for a subgroup of dystonias. Further exploration of the extent of mitochondrial dysfunction in other dystonias and of how the dysfunction might cause dystonia could provide another biological target of value to several unrelated forms of dystonia.

At the physiological level, a common theme in the dystonias involves abnormal motor learning due to aberrant neural plasticity. ${ }^{71,72}$ This theme may be most relevant to the sporadic task-specific dystonias such as writer's cramp or musician's dystonia, although there is evidence for maladaptive plasticity in the inherited gen- eralized dystonias too. ${ }^{73}$ Further studies aimed at elucidating the molecular and cellular basis for aberrant neuroplasticity could therefore provide guidance toward targets for therapy. Another common theme for multiple forms of human dystonia is an increase in cortical excitability. ${ }^{74}$ Even though the molecular basis for this phenomenon is unknown, therapies aimed at reducing cortical excitability may be useful. Many currently available antiepileptic drugs have the ability to reduce cortical excitability, but they are not often useful in the dystonias. Further studies that address the mechanisms responsible for enhanced cortical excitability are needed to guide the development of alternative drugs.

It is clear that several biochemical and physiological processes are shared by apparently unrelated subgroups of dystonia (FIG. 1D). The currently identified mechanisms are not mutually exclusive, and may even be related to each other. For example, dopaminergic neurons have an unusually high requirement for mitochondrial energy production. Thus, the dopamine and mitochondrial themes may be related at some level. Cortical excitability and neuroplasticity also are influenced by dopaminergic mechanisms, providing a potential clue to the biochemical substrates for shared physiological anomalies. It is likely that other shared mechanisms will be uncovered as further research into the pathogenesis of dystonia continues. On the other hand, the shared themes identified for specific subgroups of dystonias may not be related. It may be that they independently relate only to specific subtypes of dystonia. Better refining our understanding of these shared themes and how they may relate to each other is important for understanding the efficacy of potential new therapies targeting the common themes. Indeed, our ability to detect clinically significant benefits of new drugs may depend on a judicious selection of the most appropriate subtypes of dystonia in which to test efficacy.

\section{Empirical discovery}

Although the identification of therapeutics based on pathogenesis is scientifically sound, it is tethered to the pace of the basic research driving the mechanistic discovery. This rational approach is oftentimes slow and therefore frustrating to both patients and physicians. Another method inherent in the practices of many treating physicians is the trial-and-error strategy. Medications approved for other purposes are offered to people with dystonia, sometimes with apparent success, but often with little or no success. Still, there are some advantages to this empirical approach to drug discovery. This strategy is generally unencumbered by, and therefore not influenced by, current biases in basic mechanistic research. This opens the door to the identification of efficacious compounds that might have been overlooked in drug discovery targeted at specific pathogenic mecha- 
nisms. As a correlate, compounds identified via empirical testing have the potential to reveal novel mechanistic insight through the discovery of drugs that function via previously unexplored disease processes. Furthermore, if this nonmechanistic empirical approach is methodical, it is possible to test vast numbers of small-molecule compounds quickly and economically, thereby increasing the pace of drug discovery.

Because it is not feasible to perform large-scale drug screens using humans or even nonhuman primates, in vitro assays or in vivo testing in rodent models or simpler organisms are preferred. ${ }^{17}$ Although in vitro assays, particularly for DYT1 dystonia, ${ }^{34}$ are being developed, there is currently no in vitro test that is clearly associated with dystonic movements in humans. In vivo testing in rodent models is a viable alternative to in vitro testing. There has been an explosion in the development and characterization of rodent models of dystonia, and the field is now poised to exploit these models in drug screening.

The use of DYT1 dystonia as a prototype has spurred the development of several genetic models. Transgenic and knockin mouse strains that carry the $G A G$ deletion within the Torla gene and express mutant torsinA protein were engineered as etiological models. Both transgenic and knockin mice exhibit histological or biochemical abnormalities ${ }^{33,39,75-77}$ reminiscent of the changes observed in humans. ${ }^{57}$ Despite the etiologic resemblance to DYT1 dystonia, none of these models exhibits a motor disorder resembling dystonia. Nonetheless, all models exhibit some type of motor deficit that might prove useful in drug screening assays, including mild hyperactivity, poor performance on beam walking, or abnormal motor learning on a Rotarod apparatus. These models are obviously useful for elucidating pathogenesis in DYT1 dystonia, but the lack of the target behavior (i.e., dystonia) presents a challenge for drug development.

In contrast to the engineered genetic models, there are several rodent models that exhibit abnormal movements resembling human dystonia and also exhibit electrophysiological abnormalities consistent with dystonia. ${ }^{17,78}$ These occurred as spontaneous mutations in breeding colonies. The dystonia musculorum mouse and the dystonic rat both exhibit chronic generalized dystonia that arises in the first few weeks of life. ${ }^{79,80}$ The motor dysfunction is so severe that without heroic efforts to maintain the health of the animals, survival is limited. Because it is difficult to sustain large numbers of these animals, they are not well-suited to large-scale drug screening efforts. The $d t^{s z}$ hamster and the tottering mouse also exhibit generalized dystonia resulting from spontaneously occurring mutations. The genetic basis of the $d t^{s z}$ hamster is unknown but the mutation in the tottering mouse occurs in the Cacnala gene, ${ }^{81,82}$ a gene that is implicated in some forms of dystonia in humans. ${ }^{83,84}$ Instead of chronic debilitating dystonia, these models exhibit episodes of generalized dystonia interspersed with relatively normal interictal periods, which allow them to maintain nutrition and hygiene. ${ }^{85-87} \mathrm{De}$ spite the severity of the dystonic attacks, both $d t^{s z}$ hamsters and tottering mice have relatively normal life spans so large colonies are easy to maintain. In both strains, attacks of dystonia are easily triggered and therefore amenable to experimental manipulation, including drug screens. ${ }^{85,87}$ Indeed, the $d t^{s z}$ hamster already has been the subject of extensive drug testing. ${ }^{88}$

There are also several drug-induced models of dystonia in rodents. Microinjection of the excitatory glutamate receptor agonist kainic acid into the cerebellum produces acute generalized dystonia with sustained abnormal postures in both mice and rats. ${ }^{89,90}$ Although the dystonia is robust, this model is challenging to apply as a drug screen because it is labor-intensive. 3-Nitropropionic acid is an irreversible inhibitor of mitochondrial complex II that causes dystonia in humans as well as rodents. ${ }^{67,91,92}$ The obvious parallels with dystonia in humans are a clear advantage, so this model merits further scrutiny. Systemically administered Bay K 8644, an Ltype calcium channel agonist, causes generalized dystonia in both rats and mice. ${ }^{93,94}$ Because Bay K 8644 is simple to administer and the effect occurs in normal animals, large numbers of animals can be screened quickly. Another drug-induced model that holds promise is the unilateral 6-hydroxydopamine-lesioned rat treated with L-dopa. These animals exhibit abnormal movements and postures of the face, limbs, and trunk ${ }^{95}$ and thus may prove useful in identifying drugs for treatment of dystonia arising from basal ganglia dysfunction.

There is now an abundance of models on which to base a methodical nonmechanistic screening strategy. The success of such an approach is dependent on several related variables, including highly reproducible and clearly defined endpoints, validation, and the choice of test models.

Endpoints should be simple to evaluate, should be quantifiable, and should readily distinguish disease from normal behavior to permit the identification of drugs with modest or partial efficacy. ${ }^{17}$ The underlying assumption for these criteria is that the experimental variability is low, thus increasing signal-to-noise ratios and reducing the sample size. To facilitate the rapid throughput of drugs, endpoints should require limited technical expertise and should enable direct comparisons among drugs and models. Such criteria limit the use of analyses such as electromyography, which would require a significant level of expertise and time to screen a single compound. Finally, endpoints must be relevant to the disease.

Because dystonia is a clinical diagnosis, there are no readily adaptable biological assays for use as endpoints in animal testing; however, for models that exhibit dys- 
tonia, several reliable rating scales have been developed to assess severity. ${ }^{96}$ To provide a detailed analysis, behavioral inventories analogous to observation-based rating scales used in humans, such as the Abnormal Involuntary Movement Scale, or the Unified Dystonia Rating Scale, ${ }^{97}$ are used to discriminate dystonic motor behavior from several closely related motor syndromes, to evaluate temporal changes in the expression of dystonia, and to provide an estimate of overall frequency of target behaviors. ${ }^{93,96}$ Other scales assign a score to provide a measure of overall severity. ${ }^{87,95}$ Both types of scales have been used extensively to assess drug effects in dystonic rodents. The advantage of these rating scales is that they are easily quantified, readily applicable to any model that exhibits dystonia, have already proven useful for examining responses to potential therapeutics, and are objective if raters are blinded to treatment. Nonetheless, rating scales do require training to assure interexperimental and interrater reliability and are somewhat labor-intensive.

Models used for empirical screens must also have predictive validity. In the context of drug screening, predictive validity refers to the ability of the model to identify drugs that are likely to have therapeutic efficacy in humans. Predictive validity is based on past performance. If the model exhibits a positive response to known efficacious treatments, then it is likely to be useful for the identification of novel compounds. It is somewhat counterintuitive that models may have excellent predictive validity in drug screens but yet have little mechanistic relevance to the disorder. For example, pentylenetetrazole challenge and maximal electroshock seizures are two models with excellent predictive validity for anticonvulsive activity and are therefore widely used in empirical drug screens for novel antiepileptic compounds. ${ }^{98}$ These models have little relevance to the pathogenesis of seizure disorders, however, and thus drug response, not etiology, is paramount in selecting models for empirical drug development.

Implicit in the concept of predictive validity is that there are compounds with good efficacy in humans that are also effective in the animal model. These criteria present a conundrum for empirical drug discovery in dystonia. Small-molecule therapies are largely unsatisfactory for the treatment of dystonia in humans, and there is no small-molecule drug to serve as an unequivocal gold standard to validate models. Trihexyphenidyl, an often-prescribed anticholinergic, has modest efficacy in selected subtypes and may be useful as a starting point. The discovery of more efficacious compounds will help to refine drug screens through the identification of models with reliable predicative validity.

In light of the rapid progress in dystonia research, empirical drugs screens are now feasible. It is not necessary to wait for a perfect model that replicates all features of the human condition, if predictive power for drug efficacy is the primary goal. Because no model meets every criterion as a candidate for a drug discovery program, it is not prudent to rely on any single model. ${ }^{17}$ Instead, testing several models in tandem makes more sense: etiologic, phenotypic, and drug-induced models, singly and in combination. These models offer complementary advantages and disadvantages, which may increase the chances of identifying drugs for dystonia while also reducing the probability of false positives. As drugs are discovered and used to validate models, screening paradigms will need to adapt to accommodate models with broad applicability, and other models may be discarded.

There are hundreds of thousands of small molecules available for drug screening. It is therefore necessary to set priorities before initiating a screening program. Currently there are few small molecules effective for the treatment of dystonia, so patients would benefit enormously from drugs with even modest efficacy. It is therefore reasonable to start by screening small-molecule libraries composed of drugs that are already approved by the U.S. Food and Drug Administration (FDA) and marketed, such as the Prestwick Chemical Library (http:// www.prestwickchemical.fr/). This is essentially a methodical approach akin to the trial-and-error strategy used by physicians. Because these compounds have already been proven safe in humans, this approach circumvents problems associated with toxicity. Thus, a drug can reach patients quickly while simultaneously undergoing optimization in a medicinal chemistry program to improve efficacy. Nonetheless, even FDA-approved compounds will require rigorous preclinical trials in humans to establish efficacy before they become widely available for the treatment of dystonia.

The disadvantage of using only FDA-approved compounds is that novel chemical structures will be overlooked. Such compounds can be tested as the screening paradigm evolves and the initial pool of marketed drugs is exhausted. Of course, novel chemical structures identified via assays focused on pathogenesis can be quickly and easily incorporated into an ongoing screen, illustrating that empirical testing and rational drug design are not mutually exclusive, and can be synergistic.

\section{CONCLUSIONS: TRANSLATING DISCOVERY INTO TREATMENT}

In the past few decades, progress in both basic and clinical research has markedly advanced our understanding of the pathogenesis of dystonia. These advances have provided multiple novel insights into biological processes that could serve as potential targets for therapy. Simultaneously, there has been dramatic growth of experimental preclinical models in which potential new 
therapies can be tested. ${ }^{78}$ Many already are being exploited to identify possible new treatments.

Ultimately, research discoveries must be translated into clinical use. An infrastructure and strategy for testing promising new candidate therapies in humans is an essential ingredient for success. Similar to the situation for clinical trials in other movement disorders, such as Parkinson's disease or Huntington's disease, the clinical research effort for dystonia will depend on the development of rating instruments appropriate for measuring success. ${ }^{97}$ Likewise, success with clinical therapeutic trials in dystonia will require different study designs for symptomatic versus protective therapies. ${ }^{17}$ Unlike trials in other movement disorders with less heterogeneous pathogenesis, trials in dystonia must acknowledge etiological heterogeneity, and the logical corollary that different dystonia populations may need different treatments. Therefore, parsing the dystonias into meaningful subgroups based on shared biological processes is likely to be extremely valuable for identifying the most appropriate populations in which to test specific new therapies.

Drug discovery for dystonia was unimaginable just a few years ago. Such efforts are now feasible in light of major advances in both basic science research and our appreciation of important clinical differences among the dystonias.

Acknowledgments: This work was supported by the National Institutes of Health (Grants NS28384, NS33592, NS059645, and NS40470), the Bachmann-Strauss Dystonia and Parkinson Foundation, and the Dystonia Medical Research Foundation.

\section{REFERENCES}

1. Fahn S. The varied clinical expressions of dystonia. Neurol Clinics 1984;2:541-554.

2. Fahn S. Concept and classification of dystonia. Adv Neurol 1988; 50:1-8.

3. Defazio G. Epidemiology of primary and secondary dystonia. In: Stacy MA, editor. Handbook of dystonia. New York: Informa Healthcare USA, 2007:11-20.

4. Sanger TD, Delgado MR, Gaebler-Spira D, Hallett M, Mink JW; Task Force on Childhood Motor Disorders. Classification and definition of disorders causing hypertonia in childhood. Pediatrics 2003;111:e89-e97.

5. Kyllerman M, Bager B, Bensch J, Bille B, Olow I, Voss H. Dyskinetic cerebral palsy. I. Clinical categories, associated neurological abnormalities and incidences. Acta Pediatr Scand 1982;71: 543-550.

6. Albright AL, Barry MJ, Shafton DH, Ferson SS. Intrathecal baclofen for generalized dystonia. Dev Med Child Neurol 2001;43: $652-657$.

7. Tolosa E, Compta Y. Dystonia in Parkinson's disease. J Neurol 2006;253 Suppl 7:VII7-VII13.

8. Jankovic J, Tintner R. Dystonia and parkinsonism. Parkinsonism Relat Disord 2001;8:109-121.

9. Jankovic J. Treatment of dystonia. Lancet Neurol 2006;5:864872.

10. Albanese A, Barnes MP, Bhatia KP, et al. A systematic review on the diagnosis and treatment of primary (idiopathic) dystonia and dystonia plus syndromes: report of an EFNS/MDS-ES task force. Eur J Neurol 2006;13:433-444.

11. Balash Y, Giladi N. Efficacy of pharmacological treatment of dystonia: evidence-based review including meta-analysis of the effect of botulinum toxin and other cure options. Eur J Neurol 2004;11:361-370.

12. Tarsy D, Simon DK. Dystonia. N Engl J Med 2006;355:818-829.

13. Arce CA. Selective denervation in cervical dystonia. In: Stacy MA, editor. Handbook of dystonia. New York: Informa Healthcare USA, 2007:381-392.

14. Walker RH, Danisi FO, Swope DM, Goodman RR, Germano IM, Brin MF. Intrathecal baclofen for dystonia: benefits and complications during six years of experience. Mov Disord 2000;15:12421247.

15. Ostrem JL, Starr PA. Treatment of dystonia with deep brain stimulation. Neurotherapeutics 2008;5:319-329.

16. Pisani A, Bernardi G, Ding J, Surmeier DJ. Re-emergence of striatal cholinergic interneurons in movement disorders. Trends Neurosci 2007;30:545-553.

17. Jinnah HA, Richter A, Mink JW, et al. Animal models for drug discovery in dystonia. Expert Opin Drug Discov 2008;3:83-97.

18. Moore DJ, West AB, Dawson VL, Dawson TM. Molecular pathophysiology of Parkinson's disease. Annu Rev Neurosci 2005;28: 57-87.

19. Cookson MR. The biochemistry of Parkinson's disease. Annu Rev Biochem 2005;74:29-52.

20. Mochizuki H, Yasuda T, Mouradian MM. Advances in gene therapy for movement disorders. Neurotherapeutics 2008;5:260-269.

21. LeWitt PA, Taylor DC. Protection against Parkinson's disease progression: clinical experience. Neurotherapeutics 2008;5:210225.

22. Newman MB, Bakay RAE. Therapeutic potentials of human embryonic stem cells in Parkinson's disease. Neurotherapeutics 2008; 5:237-251.

23. Limousin P, Martinez-Torres I. Deep brain stimulation for Parkinson's disease. Neurotherapeutics 2008;5:309-319.

24. Gross R. What happened to posteroventral pallidotomy for Parkinson's disease and dsytonia? Neurotherapeutics 2008;5:281-293.

25. Segawa M, Nomura Y, Nishiyama N. Dopa-responsive dystonia In: Stacy MA, editor. Handbook of dystonia. New York: Informa Healthcare USA, 2007:219-244.

26. Jinnah HA, Hess EJ. A new twist on the anatomy of dystonia: the basal ganglia and the cerebellum. Neurology 2006;67:1740-1741.

27. McGeer EG, McGeer PL. The dystonias. Can J Neurol Sci 1988; 15:447-483.

28. Marks WJ Jr. Brain surgery for dystonia. In: Stacy MA, Handbook of dystonia. New York: Informa Healthcare USA, 2007:393-406.

29. Ozelius LJ, Hewett JW, Page CE, et al. The early-onset torsion dystonia gene $(D Y T 1)$ encodes an ATP-binding protein. Nat Genet 1997;17:40-48.

30. Goodchild RE, Dauer WT. Mislocalization to the nuclear envelope: an effect of the dystonia-causing torsinA mutation. Proc Natl Acad Sci U S A 2004;101:847-853.

31. Torres GE, Sweeney AL, Beaulieu JM, Shashidharan P, Caron MG. Effect of torsinA on membrane proteins reveals a loss of function and a dominant-negative phenotype of the dystonia-associated $\Delta$ E-torsinA mutant. Proc Natl Acad Sci U S A 2004;101: $15650-15655$.

32. Misbahuddin A, Placzek MR, Taanman JW, et al. Mutant torsinA, which causes early-onset primary torsion dystonia, is redistributed to membranous structures enriched in vesicular monoamine transporter in cultured human SH-SY5Y cells. Mov Disord 2005;20: 432-440.

33. Balcioglu A, Kim MO, Sharma N, Cha JH, Breakefield XO, Standaert DG. Dopamine release is impaired in a mouse model of DYT1 dystonia. J Neurochem 2007;102:783-788.

34. Hewett JW, Tannous B, Niland BP, et al. Mutant torsinA interferes with protein processing through the secretory pathway in DYT1 dystonia cells. Proc Natl Acad Sci U S A 2007;104:7271-7276.

35. Hewett JW, Zeng J, Niland BP, Bragg DC, Breakefield XO. Dystonia-causing mutant torsin $\mathrm{A}$ inhibits cell adhesion and neurite extension through interference with cytoskeletal dynamics. Neurobiol Dis 2006;22:98-111.

36. Pisani A, Martella G, Tscherter A, et al. Altered responses to dopaminergic D2 receptor activation and N-type calcium currents in striatal cholinergic interneurons in a mouse model of DYT1 dystonia. Neurobiol Dis 2006;24:318-325. 
37. Hewett J, Gonzalez-Agosti C, Slater D, et al. Mutant torsinA, responsible for early-onset torsion dystonia, forms membrane inclusions in cultured neural cells. Hum Mol Genet 2000;9:14031413.

38. McNaught KS, Kapustin A, Jackson T, et al. Brainstem pathology in DYT1 primary torsion dystonia [Erratum in: Ann Neurol 2004; 56:750]. Ann Neurol 2004;56:541-547.

39. Goodchild RE, Kim CE, Dauer WT. Loss of the dystonia-associated protein torsin A selectively disrupts the neuronal nuclear envelope. Neuron 2005;48:923-932.

40. Gonzalez-Alegre P, Paulson HL. Aberrant cellular behavior of mutant torsin A implicates nuclear envelope dysfunction in DYT1 dystonia. J Neurosci 2004;17:2593-2601.

41. Eidelberg D, Moeller JR, Antonini A, et al. Functional brain networks in DYT1 dystonia. Ann Neurol 1998;44:303-312.

42. Gonzalez-Alegre P, Bode N, Davidson BL, Paulson HL. Silencing primary dystonia: lentiviral-mediated RNA interference therapy for DYT1 dystonia. J Neurosci 2005;25:10502-10509.

43. Caldwell GA, Cao S, Sexton EG, Gelwix CC, Bevel JP, Caldwell KA. Suppression of polyglutamine-induced protein aggregation in Caenorhabditis elegans by torsin proteins. Hum Mol Genet 2003; 12:307-319.

44. Cao S, Gelwix CC, Caldwell KA, Caldwell GA. Torsin-mediated protection from cellular stress in the dopaminergic neurons of Caenorhabditis elegans. J Neurosci 2005;25:3801-3812.

45. McLean PJ, Kawamata H, Shariff S, et al. TorsinA and heat shock proteins act as molecular chaperones: suppression of alphasynuclein aggregation. J Neurochem 2002;83:846-854.

46. Clarimon J, Asgeirsson H, Singleton A, et al. Torsin A haplotype predisposes to idiopathic dystonia. Ann Neurol 2005;57:765-767.

47. Kamm C, Asmus F, Mueller J, et al. Strong genetic evidence for association of TOR $1 A / T O R 1 B$ with idiopathic dystonia. Neurology 2006;67:1857-1859.

48. Sibbing D, Asmus F, König IR, et al. Candidate gene studies in focal dystonia. Neurology 2003;61:1010-1097.

49. Hague S, Klaffke S, Clarimon J, et al. Lack of association with torsinA haplotype in German patients with sporadic dystonia. Neurology 2006;66:951-952.

50. Clarimon J, Brancati F, Peckham E, et al. Assessing the role of DRD5 and DYT1 in two different case-control series with primary blepharospasm. Mov Disord 2006;22:162-166.

51. Perlmutter JS, Mink JW. Dysfunction of dopaminergic pathways in dystonia. Adv Neurol 2004;94:163-170.

52. Jinnah HA, Visser JE, Harris JC, et al.; Lesch-Nyhan Disease International Study Group. Delineation of the motor disorder of Lesch-Nyhan disease. Brain 2006;129:1201-1217.

53. Visser JE, Bär PR, Jinnah HA. Lesch-Nyhan syndrome and the basal ganglia. Brain Res Brain Res Rev 2000;32:449-475.

54. Boyce S, Clarke CE, Luquin R, et al. Induction of chorea and dystonia in Parkinsonian primates. Mov Disord 1990;5:3-7.

55. Perlmutter JS, Tempel LW, Black KJ, et al. MPTP induces dystonia and parkinsonism: clues to the pathophysiology of dystonia. Neurology 1997;49:1432-1438.

56. Troiano AR, Stoessl AJ. Neuroimaging in dystonia. In: Stacy MA, editor. Handbook of dystonia. New York: Informa Healthcare USA, 2007:93-106.

57. Augood SJ, Hollingsworth Z, Albers DS, et al. Dopamine transmission in DYT1 dystonia. Adv Neurol 2004;94:53-60.

58. Cardoso F. Drug-induced dystonia. In: Stacy MA, editor. Handbook of dystonia. New York: Informa Healthcare USA, 2007:267276.

59. Nemeth AH. The genetics of primary dystonias and related disorders. Brain 2002;125:695-721.

60. Sudarsky L, Plotkin GM, Logigian EL, Johns DR. Dystonia as a presenting feature of the 3243 mitochondrial DNA mutation. Mov Disord 1999;14:488-491.

61. Head RA, de Goede CG, Newton RW, et al. Pyruvate dehydrogenase deficiency presenting as dystonia in childhood. Dev Med Child Neurol 2004;46:710-712.

62. McFarland R, Chinnery PF, Blakely EL, et al. Homoplasmy, heteroplasmy, and mitochondrial dystonia. Neurology 2007;69: 911-916.
63. Simon DK, Friedman J, Breakefield XO, et al. A heteroplasmic mitochondrial complex I gene mutation in adult-onset dystonia. Neurogenetics 2003;4:199-205.

64. Swerdlow RH, Wooten GF. A novel deafness/dystonia peptide gene mutation that causes dystonia in female carriers of MohrTranebjaerg syndrome. Ann Neurol 2001;50:537-540.

65. Kim HT, Edwards MJ, Tyson J, Quinn NP, Bitner-Glindzicz M, Bhatia KP. Blepharospasm and limb dystonia caused by MohrTranebjaerg syndrome with a novel splice site mutation in the deafness/dystonia peptide gene. Mov Disord 2007;22:1328-1331.

66. Müller-Vahl KR, Kolbe H, Egensperger R, Dengler R. Mitochondriopathy, blepharospasm, and treatment with botulinum toxin. Muscle Nerve 2000;23:647-648.

67. He F, Zhang S, Qian F, Zhang C. Delayed dystonia with striatal CT lucencies induced by a mycotoxin (3-nitropropionic acid). Neurology 1995;45:2178-2183.

68. Palfi S, Leventhal L, Goetz CG, et al. Delayed onset of progressive dystonia following subacute 3-nitropropionic acid treatment in $\mathrm{Ce}$ bus apella monkeys. Mov Disord 2000;15:524-530.

69. Schapira AH, Warner T, Gash MT, Cleeter MW, Marinho CF, Cooper JM. Complex I function in familial and sporadic dystonia. Ann Neurol 1997;41:556-559.

70. Benecke R, Strumper P, Weiss H. Electron transfer complex I defect in idiopathic dystonia. Ann Neurol 1992;32:683-686.

71. Quartarone A, Siebner HR, Rothwell JC. Task-specific hand dystonia: can too much plasticity be bad for you? Trends Neurosci 2006;29:192-199.

72. Rothwell JC, Huang YZ. Systems-level studies of movement disorders in dystonia and Parkinson's disease. Curr Opin Neurobiol 2003;13:691-695.

73. Carbon M, Ghilardi MF, Argyelan M, Dhawan V, Bressman SB, Eidelberg D. Increased cerebellar activation during sequence learning in DYT1 carriers: an equiperformance study. Brain 2008;131: $146-154$.

74. Hallett M. Pathophysiology of dystonia. J Neural Transm Suppl 2006;70:485-488.

75. Shashidharan P, Sandu D, Potla U, et al. Transgenic mouse model of early-onset DYT1 dystonia. Hum Mol Genet 2005;14:125-133.

76. Grundmann K, Reischmann B, Vanhoutte G, et al. Overexpression of human wildtype torsinA and human $\Delta$ GAG torsinA in a transgenic mouse model causes phenotypic abnormalities. Neurobiol Dis 2007;27:190-206.

77. Sharma N, Baxter MG, Petravicz J, et al. Impaired motor learning in mice expressing torsin A with the DYT1 dystonia mutation. J Neurosci 2005;25:5351-5355.

78. Jinnah HA, Hess EJ, LeDoux MS, et al. Rodent models for dystonia research: characteristics, evaluation, and utility. Mov Disord 2005;20:283-292.

79. Duchen LW. Dystonia musculorum: an inherited disease of the nervous system in the mouse. Adv Neurol 1976;14:353-365.

80. Lorden JF, McKeon TW, Baker HJ, et al. Characterization of the rat mutant dystonic (dt): a new animal model of dystonia musculorum deformans. J Neurosci 1984;4:1925-1932.

81. Fletcher CF, Lutz CM, O'Sullivan TN, et al. Absence epilepsy in tottering mutant mice is associated with calcium channel defects. Cell 1996;87:607-617.

82. Doyle J, Ren X, Lennon G, Stubbs L. Mutations in the Cacnlla4 calcium channel gene are associated with seizures, cerebellar degeneration, and ataxia in tottering and leaner mutant mice. Mamm Genome 1997;8:113-120.

83. Giffin NJ, Benton S, Goadsby PJ. Benign paroxysmal torticollis of infancy: four new cases and linkage to CACNA1A mutation. Dev Med Child Neurol 2002;44:490-493.

84. Spacey SD, Materek LA, Szczygielski BI, et al. Two novel CACNA1A gene mutations associated with episodic ataxia type 2 and interictal dystonia. Arch Neurol 2005;62:314-316.

85. Fureman BE, Jinnah HA, Hess EJ. Triggers of paroxysmal dyskinesias in the calcium channel mouse mutant tottering. Pharmacol Biochem Behav 2002;73:631-637.

86. Shirley TL, Rao LM, Hess EJ, Jinnah HA. Paroxysmal dyskinesias in mice. Mov Disord 2007;23:259-264. 
87. Löscher W, Fisher JE Jr, Schmidt D, Fredow G, Hönack D, Iturrian WB. The $s z$ mutant hamster: a genetic model of epilepsy or of paroxysmal dystonia? Mov Disord 1989;4:219-232.

88. Richter A, Löscher W. Pathology of idiopathic dystonia: findings from genetic animal models. Prog Neurobiol 1998;54:633-677.

89. Pizoli CE, Jinnah HA, Billingsley ML, Hess EJ. Abnormal cerebellar signaling induces dystonia in mice. J Neurosci 2002;22: 7825-7833.

90. Alvarez-Fischer D, Grundmann M, Lu L, Moller C, Oertel WH, Bandmann O. Gene expression studies in a novel rat dystonia model. Mov Disord 2004;19:S98 (abstract).

91. Fernagut PO, Diguet E, Stefanova N, et al. Subacute systemic 3 -nitropropionic acid intoxication induces a distinct motor disorder in adult $\mathrm{C} 57 \mathrm{Bl} / 6$ mice: behavioural and histopathological characterisation. Neuroscience 2002;114:1005-1017.

92. Ouary S, Bizat N, Altairac S, et al. Major strain differences in response to chronic systemic administration of the mitochondrial toxin 3-nitropropionic acid in rats: implications for neuroprotection studies. Neuroscience 2000;97:521-530.
93. Jinnah HA, Sepkuty JP, Ho T, et al. Calcium channel agonists and dystonia in the mouse. Mov Disord 2000;15:542-551.

94. Blake BL, Muehlmann AM, Egami K, Breese GR, Devine DP, Jinnah HA. Nifedipine suppresses self-injurious behavior in animals. Dev Neurosci 2007;29:241-250.

95. Cenci MA, Lundblad M. Utility of 6-hydroxydopamine lesioned rats in preclinical screening of novel treatments for Parkinson disease. In: LeDoux M, editor. Animal models of movement disorders. Amsterdam: Elsevier, 2005:193-208.

96. Jinnah HA, Hess EJ. The assessment of movement disorders in rodents. In: LeDoux M, editor. Animal models of movement disorders. San Diego: Elsevier Academic Press, 2005:55-71.

97. Comella CL, Leurgans S, Wuu J, Stebbins GT, Chmura T; Dystonia Study Group. Rating scales for dystonia: a multicenter trial. Mov Disord 2003;18:303-312.

98. Kupferberg HJ. Antiepileptic drug development program: a cooperative effort of government and industry. Epilepsia 1989;30 Suppl 1:S51-S56. 